\title{
Ultrastructural pathology of the 'barrier sites' in experimental autoimmune uveitis and experimental autoimmune pinealitis
}

\author{
H S Dua, A McKinnon, P G McMenamin, J V Forrester
}

\begin{abstract}
Lewis rats were immunised with retinal Santigen to induce experimental autoimmune uveitis and experimental autoimmune pinealitis. The blood-retinal and blood-pineal 'barrier sites' were examined by transmission electron microscopy. Inflammatory cell movement through Bruch's membrane involved separation of its constituent layers, cell migration through pores in the membrane, and between retinal pigment epithelial cells without causing significant displacement of the retinal pigment epithelial cell layer. The endothelial cells of retinal and pineal vessels with perivascular infiltrates showed morphological features resembling endothelium of 'high endothelial venules'.
\end{abstract}

Experimental autoimmune uveitis (EAU) induced by retinal S-antigen is a T-cell mediated autoimmune disorder and is a good model for chronic intraocular inflammation in humans. ${ }^{1}$ S-antigen immunisation also induces an autoimmune pinealitis (EAP) in experimental animals. ${ }^{2}$ The administration of an anti-S-antigen monoclonal antibody at the time of immunisation protects the animals from developing $\mathrm{EAU}^{3-5}$ but not EAP. ${ }^{45}$ This dissociation of the efferent arc of the immune response was explained by postulating that the monoclonal antibody interacted with the retinal vascular endothelium or the activated T-cells and prevented these cells from breaching the blood-retinal barrier. ${ }^{45}$ However, in the pineal gland, where the bloodbrain barrier does not exist, ${ }^{6}$ such a mechanism would not function, and the activated $\mathrm{T}$-cells would have ready access to the S-antigencontaining pinealocytes.

The exact mechanism by which inflammatory cells leave the vascular compartment at sites of inflammation is not clearly understood. Lymphocytes are known to migrate through the vascular endothelium. This process involves a step in which specific receptors on the lymphocyte cell membrane recognise corresponding molecules on the vascular endothelium, resulting in lymphocyte-endothelial adhesion. Such adhesion is an important prerequisite for lymphocyte migration across vascular endothelium. ${ }^{7}$ In lymphoid organs and certain chronically inflamed tissues extravasation of lymphocytes occurs through specialised venules known as high endothelial venules. The endothelial cells of these venules are tall and have the specialised function of recruiting circulating lymphocytes and directing them through the vessel wall into the surrounding tissue ${ }^{78}$ Antigenic stimulation, of a lymph node for example, is known to induce an increase in its high endothelial vasculature. ' High endothelial venules have been demonstrated in autoimmune lesions and in immunologically reactive tissue round tumours. ${ }^{10}$ "Whether similar mechanisms directing lymphocyte traffic exist at the bloodretinal and blood-pineal 'barrier sites' in autoimmune uveitis and pinealitis is not known.

The blood-retinal barrier consists of $(a)$ Bruch's membrane and retinal pigment epithelium (RPE) layer posteriorly, and (b) the retinal vascular endothelium with its tight junctions and lack of fenestrations, anteriorly. The pineal vessels in rats are devoid of tight junctions and are largely fenestrated. We examined these 'barrier sites' in the eyes and pineal glands of Lewis rats with EAU and EAP using transmission electron microscopy.

\section{Material and methods}

Pure bovine S-antigen was prepared by the method of Al-Mahdawi et al. ${ }^{12}$ Six female Lewis rats, 6 to 8 weeks old, were immunised by a single footpad injection of $50 \mu \mathrm{g}$ of bovine Santigen emulsified in complete Freund's adjuvant (total volume of injection was $0.1 \mathrm{ml}$ ). The animals also received an intraperitoneal injection of heat inactivated Bordetella pertussis, $10^{10}$ cells in $1 \mathrm{ml}$ of phosphate buffered saline. Fourteen to 17 days after immunisation, at the peak of the inflammatory response, the animals were killed and the eyes and pineal glands removed. Four control animals were given adjuvants without Santigen. The animals were housed and treated in compliance with the British Home Office code of practice for the housing and care of animals used in scientific procedures.

The tissues were fixed in $2.5 \%$ glutaraldehyde, embedded in epoxy resin, and processed for transmission electron microscopy (TEM) as described before. ${ }^{13}$

\section{Results}

POSTERIOR BLOOD RETINAL BARRIER

The choroid was thickened and packed with lymphocytes, monocytes, macrophages, and some polymorphs. In several animals Bruch's membrane was separated into its outer collagenous layer at the choriocapillaris and the inner cuticular layer at the RPE by the invading inflammatory cells (Figs 1A, B). Although Bruch's membrane was separated into its con- 
Figure $1 A$ Separated layers of Bruch's membrane (broad arrows) with inflammatory cells between the layers. RPE cells (long arrow with mononuclear cells (arrow heads) adjacent to it. (Bar $=7000 \mathrm{~nm}$.)

Figure 1B Inflammatory cells (arrows) lying beneath and between RPE cells. $($ Bar $=4000 \mathrm{~nm}$.)
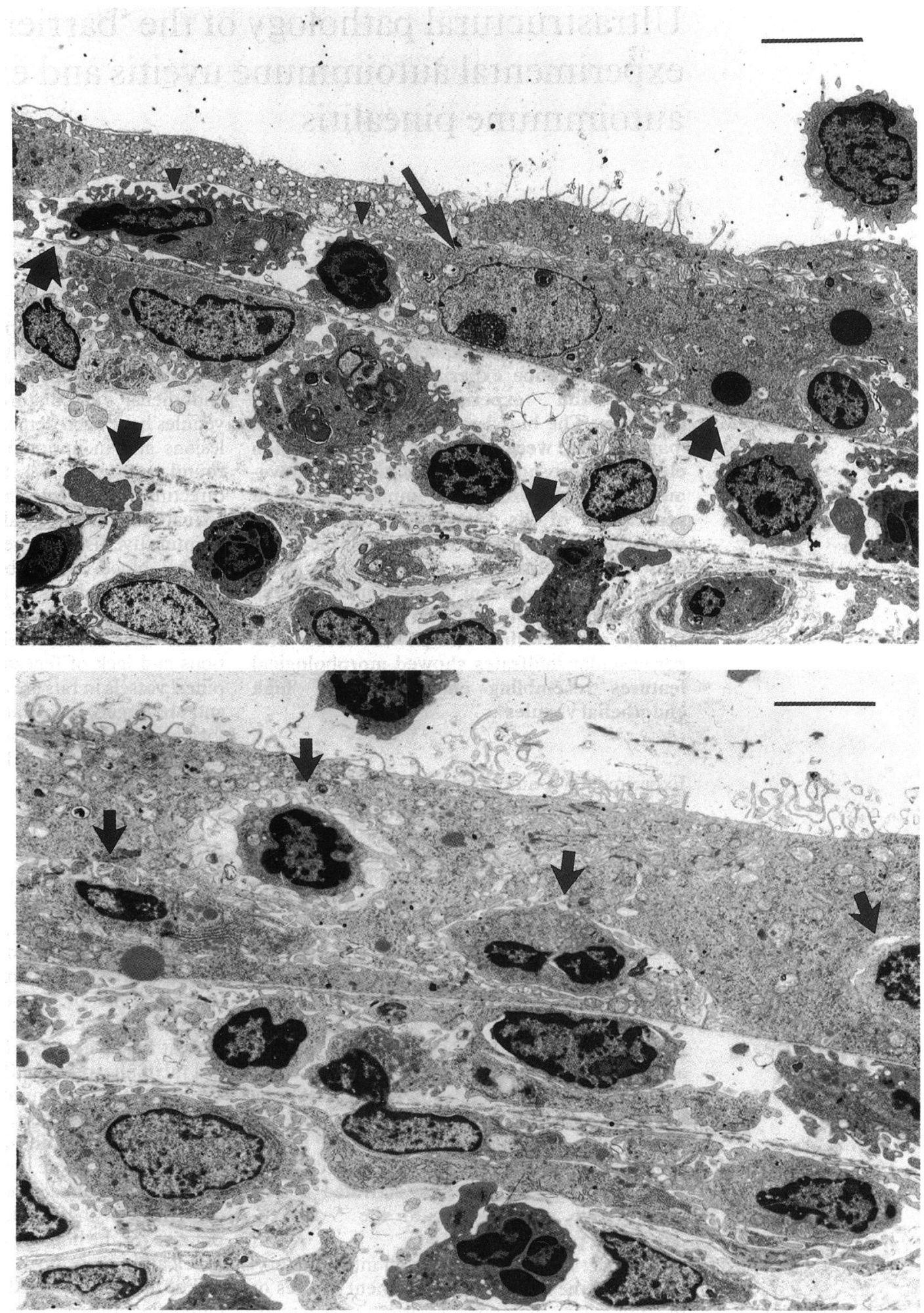

stituent components, it remained intact, and inflammatory cells penetrated though pores in the collagenous layers (Fig 1C). The inflammatory cells observed between layers of Bruch's membrane were macrophages, monocytes, lymphocytes, and occasional polymorphs. From Bruch's membrane inflammatory cells passed through the inner cuticular layer and migrated beneath and between the RPE cells (Fig 1B), eventually making their way into the subretinal space (photoreceptor layer). The cells in the subretinal space were predominantly monocytes, lymphocytes, macrophages, and some polymorphs showing evidence of phagocytosis of rod outer segment debris (Fig 1D). In some places these cells formed focal aggregates (Fig lE). The focal lesions were composed mainly of lymphoid cells with occasional macrophages showing phagosomes. The RPE cells showed stunted or absent processes but remained well preserved and maintained their monolayer distribution even in advanced EAU (Fig 1F). The endothelial cells of the choroid showed a normal flat morphology.

\section{ANTERIOR BLOOD RETINAL BARRIER}

The perivascular infiltrate was predominantly 


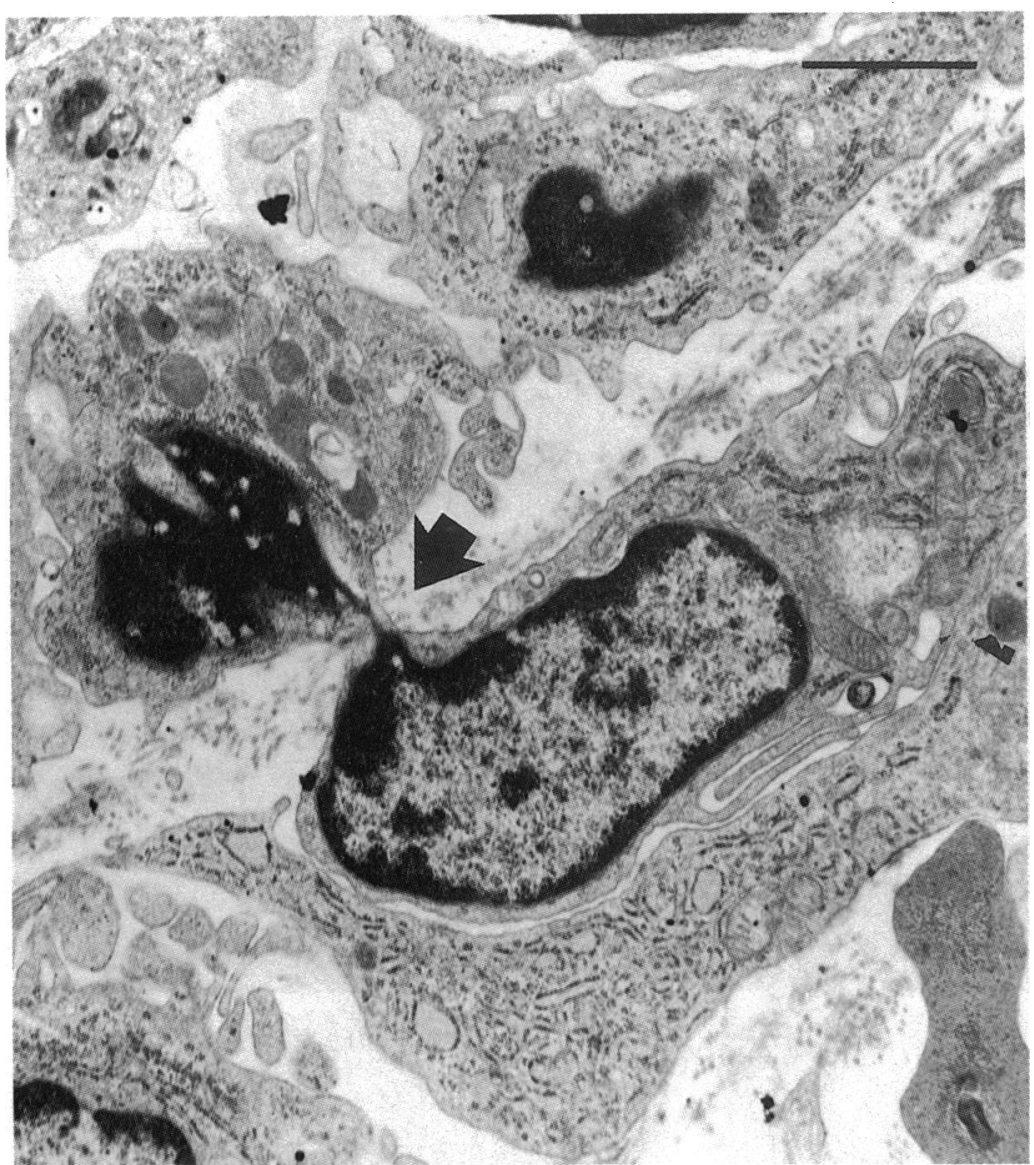

Figure 1C Mononuclear cell (macrophage) migrating through a pore (arrow) in the separated collagenous layer of Bruch's membrane. (Bar=1300 nm.)

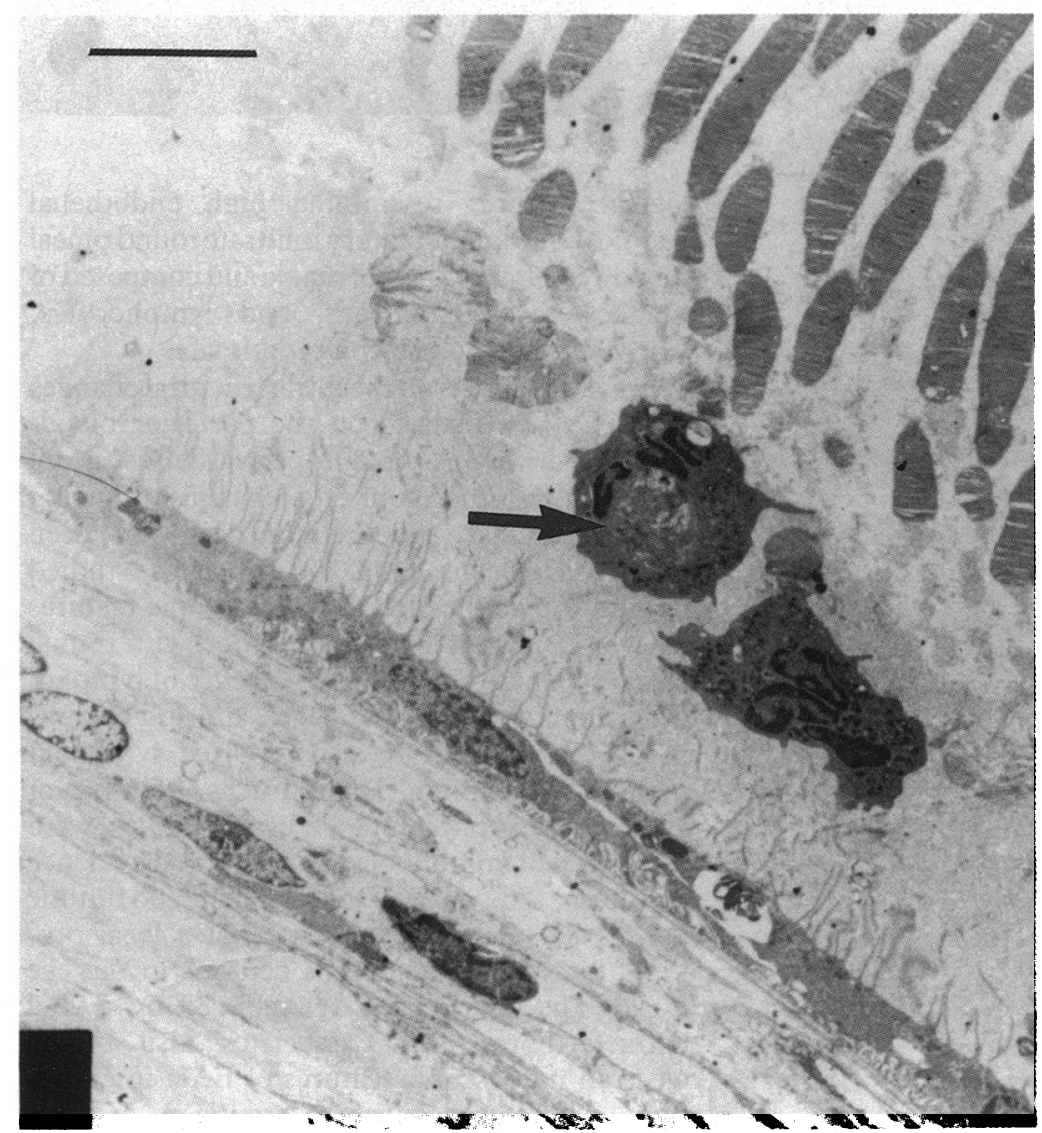

Figure 1D Two polymorphonuclear cells lying in the subretinal exudate with evidence of phagocytosis of rod outer segment debris (arrow). (Bar $=12000 \mathrm{~nm})$. mononuclear showing monocytes, macrophages, and lymphocytes in approximately equal proportions. In addition, as in the choroid, there were some polymorphonuclear cells as well. A large number of inflammatory cells could be seen in the vitreous on one side and extending through the layers of the retina on the other. Inflamed vessels showed tall, plump endothelial cells with irregular pale nuclei containing dense peripheral heterochromatin. The cytoplasm showed prominent rough endoplasmic reticulum, a large number of ribosomes and mitochondria, and occasional large electron dense bodies (Figs 2A, B). The tight junctions of the endothelial cells were found to be intact in all the vessels studied (Fig 2B). At places, platelet thrombi were seen in the retinal vessels. These vessels had flat endothelial cells and showed little evidence of perivascular inflammation.

Control animals showed normal morphology without any evidence of inflammatory cell infiltration in the choroid, subretinal, or perivascular spaces.

\section{PINEAL BLOOD VESSELS}

The inflammatory exudate in the pineal gland was haemorrhagic and exclusively mononuclear, being composed entirely of monocytes, macrophages and lymphocytes (Figs 3A, B). Unlike the retinal exudate there were no polymorphonuclear cells. Almost all lesions were related to blood vessels. Some of the blood vessels were packed with mononuclear cells. The endothelial cells showed similar features to the tall endothelial cells in the retina (Figs 3A, B). All control tissues showed normal morphology.

\section{Discussion}

In the present study we immunised Lewis rats with retinal S-antigen and adjuvant to induce experimental autoimmune uveitis and pinealitis. The animals were killed at the peak of inflammatory response and the eyes and pineal glands studied by transmission electron microscopy. The blood-retinal and blood-pineal 'barrier sites' were studied in detail. The posterior blood retinal barrier site showed splitting of Bruch's membrane and inflammatory cell migration through pores in the split layers. These cells were seen to lie between layers of Bruch's membrane and beneath the RPE cell layer. The RPE retained its monolayer configuration but showed loss of cell processes. Subretinal inflammatory cells were either dispersed in the subretinal fluid or formed focal granulomatous lesions. These cells were monocytes, macrophages, lymphocytes, and occasional polymorphs. The anterior blood retinal barrier - that is, the retinal blood vessels - showed perivascular infiltration with monocytes, macrophages, lymphoyctes, and polymorphs. The endothelium of the inflamed vessels showed morphological features resembling high endothelial venules. Pineal inflammation was manifest as multiple foci of perivasculitis, particularly in the subcapsular region and at the junction of the pineal stalk with the body of the gland. The endothelium of inflamed pineal vessels also showed morpho- 
Figure $1 E$ Focal lesion between Bruch's membrane (large arrow, right) and photoreceptor nuclei (small arrows, left $).($ Bar $=$ $7000 \mathrm{~nm}$.)

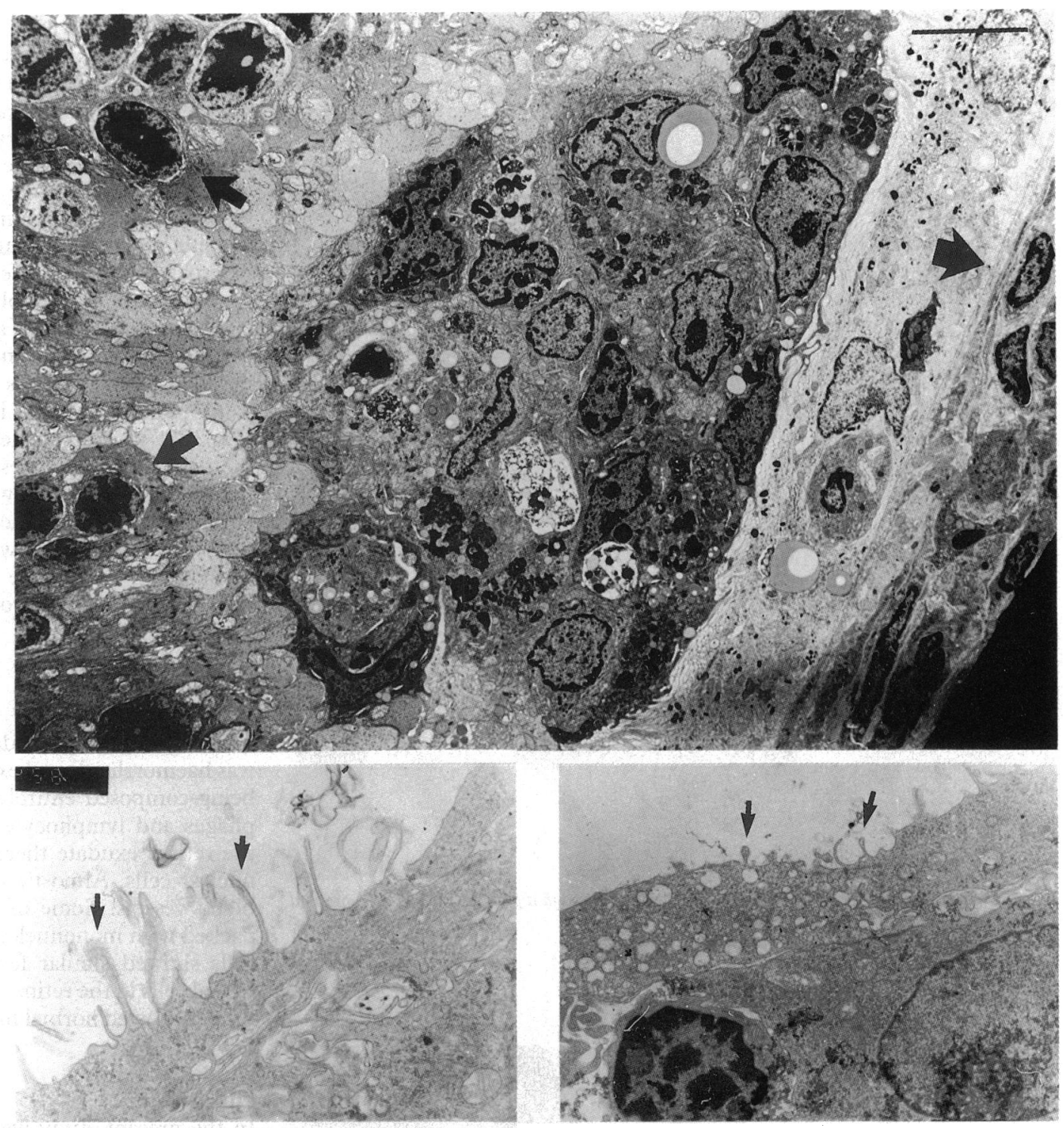

logical features resembling high endothelial venules. The inflammatory infiltrate round pineal blood vessels was haemorrhagic and composed of monocytes, macrophages, and lymphocytes. There were no polymorphs at this site.

Previous studies on the ultrastructural changes in EAU have focused on the posterior bloodretinal barrier. Although perivasculitis is an early feature of EAU in rats, changes occurring in the vascular endothelium have not been described in detail. Faure and associates ${ }^{14}$ studied in guinea pigs the electron microscopic features of uveitis induced by immunisation with retinal extracts. They observed the presence of lymphocytes, monocytes, macrophages, and polymorphs in the choroid. They also reported lymphoid nodules resembling Dalen-Fuchs nodules of sympathetic ophthalmia. These nodules were composed of immunoblasts and showed evidence of mitosis. The presence of blast cells and mitotic figures led them to postulate that a local proliferation of lymphoid cells ocurred in the eye during the course of EAU induced by retinal extracts. Forrester and associates ${ }^{13}$ also studied electron microscopic features of EAU in guinea pigs. They used purified S-antigen in their model and reported an early appearance of isolated macrophages among the disrupting rod
Figure 2A Retinal vasculitis, tall endothelial cell occupying most of the vessel lumen (large arrow). Note the large dense cytoplasmic body (small arrow) and irregular nucleus. (Bar $=4000$ nm.) 
Figure 2B Two tall endothelial cells in a retinal vessel (large arrows). Note the irregular pale nucleus and abundant rough endoplasmic reticulum. The cell junction is intact (arrow head). A macrophage

(curved arrow) lies adjacent to the basement membrane. $(B a r=800 \mathrm{~nm}$. $)$

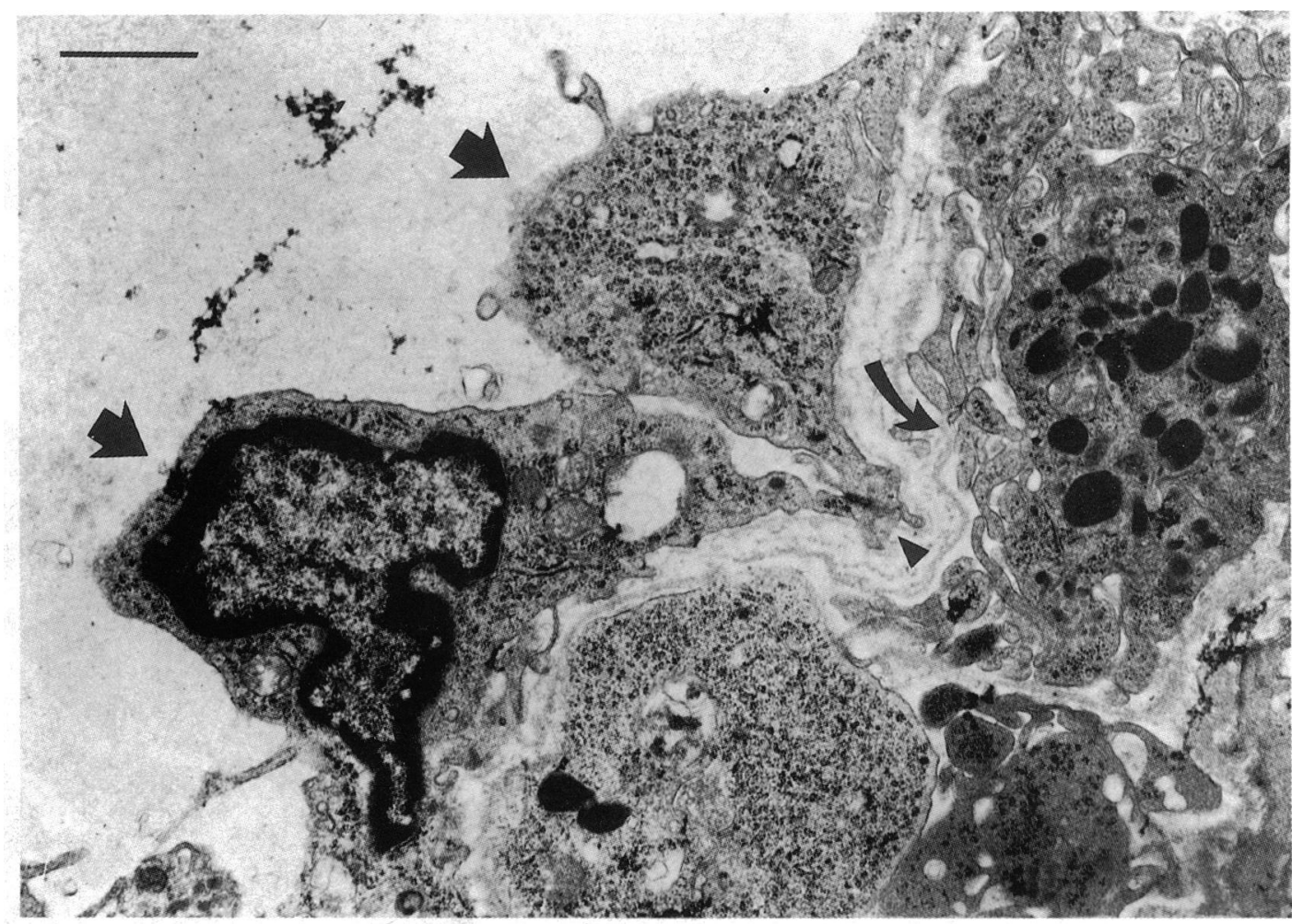

unlike the rat (and human) retina, is devoid of blood vessels. Changes occurring in and around retinal blood vessels could therefore not be studied.

De Kozak et $a l^{15}$ studied the electron microscopic features of a hyperacute form of EAU in Lewis rats. They too used whole retinal extracts obtained from guinea pig or rat eyes to induce EAU. They reported the invasion of capillary walls by polymorphonuclear cells but have not commented on the state of the vascular endothelium. None of the previous studies have documented the ultrastructural features of the associated pineal inflammation or made any attempt to explain the mechanism of inflammatory cell migration across the blood-retinal barrier.

Lymphocyte migration is an important prerequisite for the proliferation, differentiation, and selective distribution of antigen specific cells. The migration of lymphocytes through the vascular endothelium involves specific lymphocyte-endothelium adhesion mechanisms that direct lymphocytes from the blood stream into lymphoid organs or inflamed tissues. In lymphoid organs migration occurs through a specialised vascular endothelium of postcapillary venules, called high endothelial venules (HEVs). ${ }^{7-9}$ Lymphocytes have been shown to bind selectively to the endothelium of HEVs via specific receptors known as lymphocyte homing receptors. Lymphocyte homing receptors recognise and specifically bind to corresponding receptors on the endothelium of HEVs. These endothelial adhesion receptors are known as 'vascular addressins'. ${ }^{7}$ HEVs are normally found in all secondary lymphoid tissues except the spleen. However, HEV-like structures are known to develop in chronic inflammatory conditions, including autoimmune lesions, and in cell mediated immune reactions in the skin Surrounding haemorrhagic, mononuclear exudate.

outer segments. This was followed by a massi infiltration with mononuclear cell cells were also present in the infiltrate.

Both these studies differed significantly from the present study because the guinea pig retina, 
Figure 3B Pineal vasculitis, several tall endothelial cells (arrow heads, centre) with a perivascular haemorrhagic mononuclear exudate. $($ Bar $=10000 \mathrm{~nm}$.)

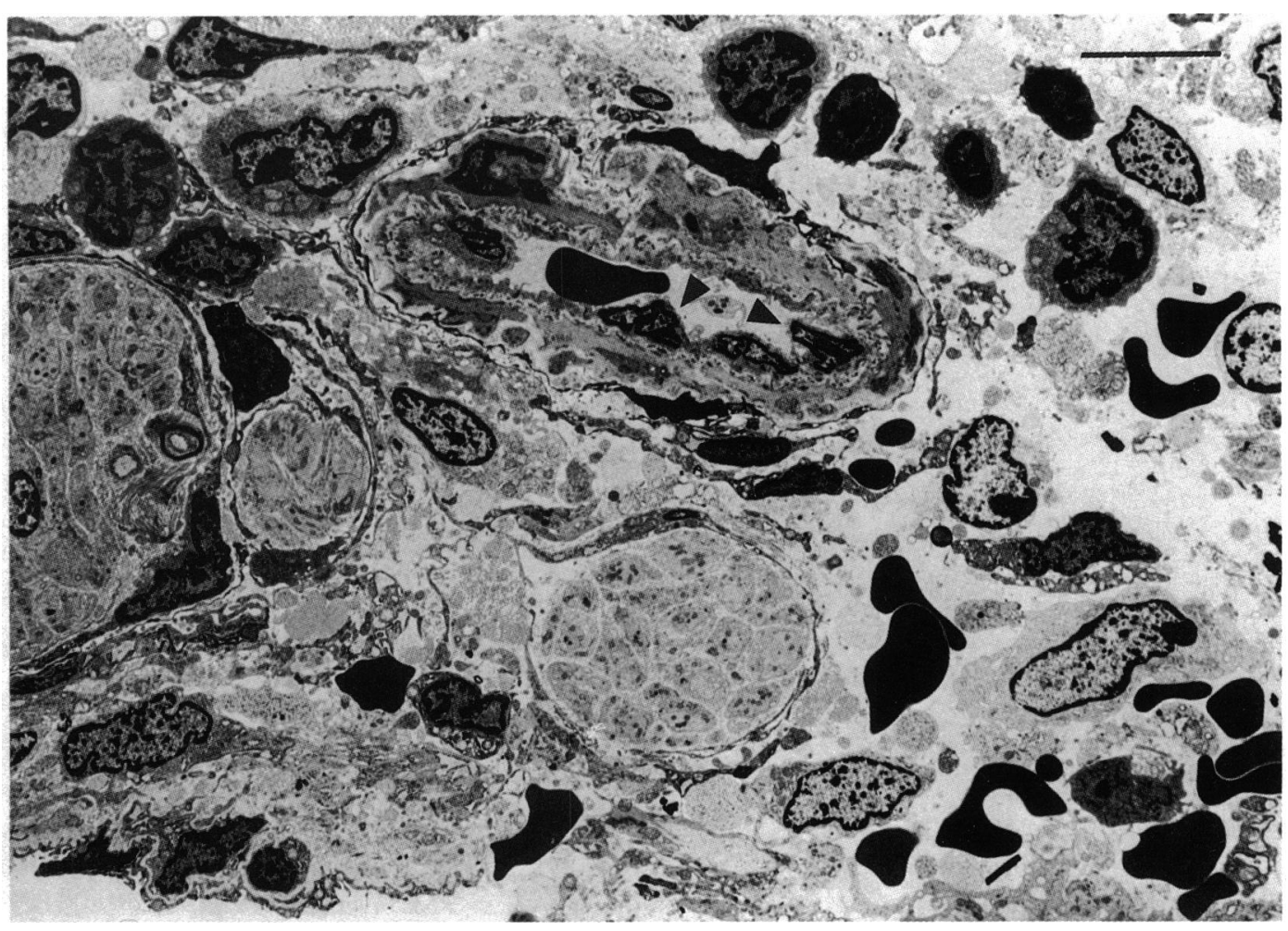

and synovial membrane. ${ }^{10}$ The endothelium of HEVs is cylindrical or cuboidal in shape. They contain electron dense organelles, numerous free and clustered ribosomes, and abundant rough endoplasmic reticulum. These cells also show occasional large dense bodies in the cytoplasm, unlike other endothelial cells. The nuclei are large, pale, and irregular. The chromatin is loose centrally and condensed at the periphery. ${ }^{89}$

In the present study all retinal blood vessels with perivascular infiltrates showed altered endothelial cells with some morphological features similar to the endothelial cells of HEVs. The endothelial cells were plump, cuboidal in shape, with prominent rough endoplasmic reticulum and a large number of ribosomes and mitochondria. Some of them also showed large electron dense bodies in the cytoplasm. These changes may be related to the recruitment and migration of lymphocytes across the blood retinal barrier.

How inflammatory cells cross the blood-retinal barrier is unclear. The obvious path, as suggested by Duijvestijn and Hamann, ${ }^{7}$ would be through the endothelial cell junctions. We did not observe any breach in the junctions of endothelial cells in any of the HEV-like vessels studied. On the contrary, the junctions between high endothelial cells seemed to be well preserved, even though inflammatory cells were seen immediately outside the vessel wall. It is possible that in retinal vessels the cell junctions temporarily open to permit lymphocyte exodus and rapidly re-form after the cell has passed through. Migration of lymphocytes through the endothelial cell (transcytosis) may be an alternative mechanism.

Although the choroid showed a considerable amount of inflammatory cell infiltrate, the endothelial cells of choroidal vessels did not show any of the changes observed in the retinal vascular endothelium. This would suggest that vascular endothelial cell participation in the inflammatory process at this site is different from that in the retinal vessels.

In previous studies ${ }^{14}$ is on the ultrastructural pathology of EAU it is suggested that the migration of inflammatory cells from choroid to the subretinal space occurs through breaks or defects in the RPE/Bruch's membrane layer. This does not appear to be essential, as illustrated in this study, where inflammatory cells were shown to migrate through pores in the Bruch's membrane which was separated into two layers with inflammatory cells lying in between. From here cells migrated to lie beneath and between RPE cells leaving the RPE layer essentially intact. Subretinal focal lesions were observed on intact Bruch's membrane indicating that the migration of cells from the choroid to this site is more complex than a mere influx of cells through a breach in the RPE/Bruch's membrane layer. The use of retinal extracts rather than S-antigen in the previous studies may have induced a more severe form of EAU with destruction of the RPE/ Bruch's membrane layer.

The pineal inflammatory response was essentially a perivasculitis involving predominantly vessels located in the subcapsular region and at the junction of the pineal stalk with the body of the gland. Like retinal vessels, the pineal vascular endothelium showed features of increased metabolic activity and a morphological resemblance to HEVs. The pineal inflammatory exudate differed from the retinal exudate in two respects. There were a large number of red blood cells and a striking lack of polymorphonuclear cells in the pineal exudate. The presence of red blood cells in the pineal perivascular exudate would indicate increased fragility and ready extravasation at this site. In spite of this the absence of polymorphonuclear cells from the 
exudate would suggest that specific mechanisms which are involved in the recruitment of these cells operate in the retina but not in the pineal gland. As in retinal vessels, endothelial cell junctions of pineal vessels remained intact, even though the cell morphology was considerably altered. However, at this site inflammatory cell migration through fenestrations in the endothelial lining was more likely.

The differences in the vascular endothelial response between the pineal and retinal vessels on the one hand and the choroidal vessels on the other, and the differences in the composition of the inflammatory exudate in the eye and pineal gland during the course of an inflammation induced by a common antigen, S-antigen, would suggest that more than one mechanism is involved in the recruitment and migration of antigen specific lymphocytes and that these mechanisms are, to some extent, specific to the tissue involved. Surface receptors (vascular addressins) expressed by the endothelial cells of inflamed vessels, which interact with lymphocyte homing receptors, may have an important role in determining the nature of inflammatory exudate in the eye and pineal gland. Differences in the inflammatory cell infiltrate between eye and pineal gland may be due to expression of different receptors (addressins) at these sites. If it can be demonstrated that specific surface receptors (vascular addressins) are in fact expressed on the endothelium of retinal and pineal vessels in EAU and EAP, it may prove to be an important mechanism in the recruitment of activated antigen specific lymphocytes in these autoimmune disorders.
This work was supported by a grant from the Grampian Health Board, Aberdeen, Scotland.

1 Forrester JV, Liversidge J, Dua HS, Towler H, Mcmenamin PG. Comparison of clinical and experimental uveitis. Curr EG. Comparison of clinical and 1990 ; 9 (suppl): 75-84.

2 Kalsow CM, Wacker WB. Pineal gland involvement in retinainduced experimental allergic uveitis. Invest Ophthalmol Vis Sci 1978; 17: 774-83.

3 De Kozak Y, Mirshahi M, Boucheix C, Faure JP. Inhibition of experimental autoimmune uveoretinitis in rats by $S$-antigen specific monoclonal antibodies. Eur $\mathcal{F}$ Immunol 1985; 15: 1107-11.

4 Dua HS, Sewell HF, Forrester JV. The effect of retinal Santigen specific monoclonal antibody therapy on experimental autoimmune uveoretinitis and experimental autoimmune pinealitis. Clin Exp Immunol 1989; 75: 100-5.

5 Dua HS, Liversidge J, Forrester JV. Immunomodulation of experimental autoimmune uveitis using a rat anti retinal Santigen specific monoclonal antibody: evidence for species difference. Eye 1989; 3: 69-78.

6 Haymaker W, Liss L, Vogel FS, Johnson JE Jr, Adams RD, Scharenberg $R$. The pineal gland. In: Haymaker W, Adams $\mathrm{RD}$, eds. Histology and histopathology of the nervous system. Springfield: Thomas, 1982; 2: 1801-2023.

7 Duijvestijn A, Hamann A. Mechanisms and regulation of lymphocytes migration. Immunol Today 1989; 10: 23-8.

$8 \mathrm{Kraal}$ G, Duijvestijn A, Hendriks HH. The endothelium of the high endothelial venule: a specialised endothelium with unique properties. Exp Cell Biol 1987; 55: 1-10.

9 Anderson ND, Anderson AO, Wyllie RG. Specialised structure and metabolic activities of high endothelial venules in rat lymphatic tissues. Immunology 1976; 31: 455-73.

10 Freemont AJ, Jones CJB, Bromley M, Andrews P. Changes in vascular endothelium related to lymphocyte collections in diseased synovia. Arthritis Rheum 1983; 26: 1427-33.

11 Freemont AJ. The small blood vessels in areas of lymphocyte infiltration around malignant neoplasms, $\mathrm{Br} \mathcal{F}$ Cancer 1982; 46: 283-8.

12 Al-Mahdawi S, Forrester JV, Lee WR. A simplified method for the isolation of highly purified bovine retinal S-antigen. I Neuroimmunol 1987; 14: 99-108.

13 Forrester JV, Borthwick GM, McMenamin PG. Ultrastructural pathology of S-antigen uveoretinitis. Invest Ophthalmol Vis Sci 1985; 26: 1281-92.

14 Faure JP, Dorey C, Tuyen VV, de Kozak Y. Experimental autoimmune uveo-retinitis and specificity of retinal antigens. In: Streiff EB, ed. Modern problems in ophthalmology. Basel: Karger, 1976; 16: 21-9.

15 De Kozak Y, Thillaye B, Renard G, Faure JP. Hyperacute form of experimental autoimmune uveo-retinitis in Lewis rats; electron microscopic study. Graefes Arch Clin Exp Ophthalmol 1978; 208: 135-42. 